\title{
Efeitos da posição dos joelhos no plano frontal sobre as forças aplicadas no ciclismo: estudo preliminar
}

\author{
Effects of knee frontal plane position on pedal forces during \\ cycling: A preliminary study
}

Rodrigo Rico Bini ${ }^{1}$

Felipe Pivetta Carpes 2

Fernando Diefenthaeler ${ }^{2}$

1 Institute of Sport and Recreation Research New Zealand. School of Sport and Recreation. Auckland University of Technology. North Shore, Auckland, New Zealand.

2 Universidade Federal do Rio Grande do Sul. Laboratório de Pesquisa do Exercício. Grupo de Estudo e Pesquisa em Ciclismo. Porto Alegre, RS. Brasil.

Recebido em 18/01/08 Revisado em 26/05/08 Aprovado em 11/11/08
Resumo - O objetivo foi comparar a força aplicada no pedal de ciclistas, pedalando com os joelhos tangenciando o quadro da bicicleta (posição de Adução), com a posição preferida (posição de Referência), e a posição com os joelhos afastados do quadro da bicicleta (posição de Abdução). Seis ciclistas foram avaliados. Foi determinado o consumo máximo de oxigênio $\left(\mathrm{VO}_{2 \mathrm{Máx}}\right)$, sendo posteriormente os ciclistas avaliados em três situações: Posição de Referência; Posição de Adução; Posição de Abdução. O VO, referente ao segundo limiar ventilatório foi utilizado para normalização da carga de trabalho. A medição da força aplicada foi realizada por um pedal instrumentado. $\mathrm{O} \mathrm{VO}_{2}$ e a potência produzida não diferiram entre as posições de Adução (50,4 $\pm 6,9 \mathrm{ml}^{\mathrm{kg}}{ }^{-1} \mathrm{~min}^{-1}$ e $263 \pm 29$ W), Abdução (50,8 $\pm 5,9$ $\mathrm{ml} \cdot \mathrm{kg}^{-1} \cdot \mathrm{min}^{-1}$ e $\left.250 \pm 46 \mathrm{~W}\right)$ e a posição de Referência $\left(50,4 \pm 5,9 \mathrm{ml} \cdot \mathrm{kg}^{-1} \cdot \mathrm{min}^{-1}\right.$ e $246 \pm 47$ W). A cadência de pedalada aumentou significativamente nas posições de Adução (94 \pm $8 \mathrm{rpm})$ e Abdução (95 $\pm 5 \mathrm{rpm}$ ), comparadas à Posição de Referência ( $89 \pm 8 \mathrm{rpm}$ ). Não foram observadas diferenças para a força efetiva e índice de efetividade entre as posições de Referência, Adução e Abdução. A força resultante apresentou aumento na Posição de Adução $(284,5 \pm 44,5 \mathrm{~N})$ quando comparada à Posição de Referência $(246,9 \pm$ 39,2 N). Estes resultados indicam que os ciclistas avaliados foram capazes de aplicar mais força no pedal na posição de Adução, no entanto, não foram capazes de direcionar esta força de forma mais efetiva para o movimento.

Palavras-chave: Ciclismo; Força; Joelho; Membro Inferior.

Abstract - The purpose of this study was to compare the force applied to the pedal when cycling with the knees almost touching the bicycle frame (adduction position) compared to the position usually adopted by cyclists (reference position) and a position of the knees away from the bicycle frame (abduction position). Six cyclists were evaluated. Maximal oxygen uptake $\left(\mathrm{VO}_{2 \mathrm{MAX}}\right)$ was defined, and then the three different knee positions on the frontal plane were assessed. Three minutes of cycling in the reference position, adduction position and abduction position were conducted, using oxygen uptake $\left(\mathrm{VO}_{2}\right)$ at which the cyclists had achieved the second ventilatory threshold. An instrumented two-dimensional pedal was used to measure the forces applied to the right pedal. The average $\mathrm{VO}_{2}$ and power output did not differ between the adduction $(50.4 \pm 6.9$ $\mathrm{ml}^{\mathrm{kg}} \mathrm{min}^{-1}$ and $\left.263 \pm 29 \mathrm{~W}\right)$, abduction $\left(50.8 \pm 5.9 \mathrm{mlkg}^{-1} \cdot \mathrm{min}^{-1}\right.$ and $\left.250 \pm 46 \mathrm{~W}\right)$ and reference position $\left(50.4 \pm 5.9 \mathrm{mlkg}^{-1} \mathrm{~min}^{-1}\right.$ and $\left.246 \pm 47 \mathrm{~W}\right)$. Pedaling cadence increased significantly during adduction $(94 \pm 8 \mathrm{rpm})$ and abduction $(95 \pm 5 \mathrm{rpm})$ compared to the reference position $(89 \pm 8 \mathrm{rpm})$. The force results indicated no differences in the effective force or effectiveness index between the reference, adduction and abduction positions. Resultant force increased during adduction $(284.5 \pm 44.5 \mathrm{~N})$ compared to the reference position $(246.9 \pm 39.2 \mathrm{~N})$. The results indicate that the cyclists were able to generate higher resultant force during cycling with the knees close to the bicycle frame, but were unable to effectively transmit this force to the movement.

Key words: Bycling; Force; Knee; Lower Extremity. 


\section{INTRODUÇÃO}

O desempenho no ciclismo competitivo é altamente dependente da redução da força de arrasto e do aumento das forças aplicadas nos pedais. A força de arrasto gerada pelo contato do conjunto ciclistabicicleta com o ar resulta no aumento da resistência frontal, principalmente, em altas velocidades ${ }^{1}$. Sabendo que existe uma relação direta entre a força de arrasto e o custo energético, a redução desta força resulta em otimização do desempenho do ciclista ${ }^{2,3}$.

O posicionamento do corpo tem sido um dos aspectos mais estudados na tentativa de minimizar os efeitos da resistência do ar durante o ciclismo. McCole et al. ${ }^{2}$ observaram que o uso de acessórios aerodinâmicos (ex. rodas e quadros com desenho aerodinâmico), assim como pedalar atrás de outro ciclista, reduz o custo energético. Hagberg e McCo$\mathrm{le}^{1}$ também sugerem que a otimização de aspectos aerodinâmicos pode comprometer algumas variáveis fisiológicas, afetando o desempenho no ciclismo.

Welbergen e Clijsen ${ }^{3}$ observaram uma redução de 4,5\% na potência máxima produzida durante teste submáximo de ciclismo, no qual os atletas assumiram uma posição aerodinâmica (mãos posicionadas na parte baixa do guidão), quando comparados à posição convencional de ciclismo (mãos posicionadas na parte superior do guidão). Os autores indicam, no entanto, que apesar da redução da potência em teste estacionário, a posição aerodinâmica é capaz de gerar reduções na área frontal do ciclista e conseqüentemente, na força de arrasto ${ }^{4}$.

Nesta perspectiva, outra estratégia comumente utilizada pelos ciclistas com o intuito de reduzir a força de arrasto por meio da redução na área frontal é pedalar com os joelhos quase tocando o quadro da bicicleta ${ }^{5}$. No entanto, além das possíveis implicações desta modificação postural sobre a força de arrasto, esta pode repercutir sobre a força propulsiva aplicada pelo ciclista na bicicleta. As possíveis alterações na força propulsiva poderiam ser atribuídas a uma possível mudança no direcionamento da força aplicada no pedal, como ilustra a Figura 1. Desta forma, torna-se importante a medição das componentes da força aplicada no pedal, ou mesmo da força que gera propulsão sobre a bicicleta (força efetiva) quando o ciclista pedala com os joelhos próximos ao quadro da bicicleta.

Estudos envolvendo a técnica da pedalada podem ser realizados com o uso de pedais instrumentados com transdutores de força, adaptados para a medição das componentes da força aplicada sobre o pedal, tornando possível o cálculo da força

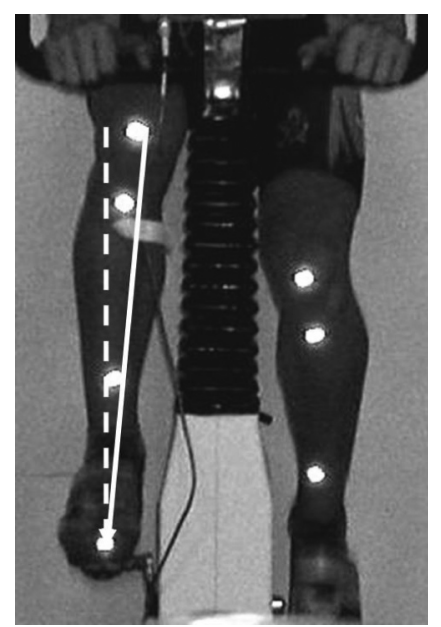

Figura 1. Ilustração do possível direcionamento da força aplicada no pedal, no plano frontal, quando alterada a posição do joelho fazendo com que este tangencie o quadro da bicicleta durante o ciclo de pedalada (Posição de Adução). O efeito da Posição de Adução sobre a força resultante no pedal é representado na figura pelo vetor branco contínuo, enquanto a posição que leva a um alinhamento ideal da força aplicada no pedal durante a pedalada é representada pelo vetor branco tracejado.

efetiva ${ }^{6}$. No entanto, nenhum estudo reportado na literatura científica foi desenvolvido, até o presente momento, com o intuito de avaliar as implicações da pedalada com os joelhos tangenciando o quadro da bicicleta sobre a força efetiva. A mudança na postura dos membros inferiores, devido à realização de uma adução do quadril, para que seja possível tangenciar com os joelhos o quadro da bicicleta, possivelmente altera o comprimento dos músculos que cruzam a articulação do quadril, afetando a sua capacidade de produção de força. Nesta perspectiva, Ericson et al. ${ }^{7}$ avaliaram seis indivíduos não-atletas pedalando com os joelhos tangenciando o quadro da bicicleta, observando aumento na componente médio-lateral da força na articulação tíbio-femoral. No entanto, os resultados não apresentam informações referentes às componentes da força normal (Fy) e tangencial $(\mathrm{Fx})$ aplicadas no pedal.

Sendo assim, o objetivo do presente estudo foi comparar a força aplicada no pedal quando os ciclistas adotam a posição com os joelhos tangenciando o quadro da bicicleta durante a pedalada (Posição de Adução) à sua posição usual de pedalada (Posição de Referência). Estas foram ainda comparadas com uma condição na qual o ciclista pedalava com os joelhos o mais afastado possível do quadro da bicicleta (Posição de Abdução). A Posição de Abdução foi avaliada com o objetivo de simular uma condição no qual os ciclistas não estivessem acostumados a pedalar. A hipótese do presente estudo foi de que as mudanças na posição 
dos joelhos em relação ao quadro da bicicleta (posições de Adução e Abdução), devido à mudança no ângulo do quadril, poderiam reduzir a força aplicada no pedal, comparado à Posição de Referência. Esta hipótese se baseia na proposta teórica apresentada na Figura 1, assim como nos resultados apresentados por Ericson et al. ${ }^{7}$ onde um aumento na componente médio-lateral da força na articulação tíbio-femoral foi observado quando se aproximou os joelhos do quadro da bicicleta. Este resultado sugere uma possível mudança nas componentes da força aplicada no pedal com a mudança na posição dos joelhos.

\section{PROCEDIMENTOS METODOLÓGICOS}

\section{Sujeitos}

Seis ciclistas de nível nacional foram voluntários a participar do estudo, o qual foi previamente aprovado pelo Comitê de Ética em Pesquisa com Seres Humanos da Instituição no qual foi realizado (protocolo número 2004311). A média e o desvio padrão dos dados referentes à idade, massa corporal, consumo máximo de oxigênio $\left(\mathrm{VO}_{2 \mathrm{Max}}\right)$, pico de potência e potência relativa à massa corporal dos sujeitos foram de: 29 anos \pm 9 anos; $71,9 \pm 4,3 \mathrm{~kg}$; 58 $\pm 9,4 \mathrm{ml}^{\mathrm{kg}}{ }^{-1} \cdot \mathrm{min}^{-1} ; 384 \pm 53 \mathrm{~W}$; e $5,53 \pm 0,9 \mathrm{~W} \cdot \mathrm{kg}^{-1}$, respectivamente. As características dos sujeitos avaliados são similares àquelas descritas previamente na literatura para ciclistas de nível nacional ${ }^{8}$.

\section{Determinação do $\mathrm{VO}_{2 \text { Máx }}$}

No primeiro dia de avaliação, foi realizado um teste com carga incremental em ciclo ergômetro estacionário $\left(\mathrm{CardiO}_{2}\right.$, Medical Graphics Corp., St. Louis, EUA), adaptado com selim, pedais e guidão similares aos utilizados em bicicletas de competição, com o objetivo de determinar o consumo máximo de oxigênio $\left(\mathrm{VO}_{2 \text { Max }}\right)$ dos ciclistas. $O$ teste incremental consistiu na utilização de uma carga inicial de $50 \mathrm{~W}$, com incrementos de $25 \mathrm{~W}$ a cada minuto 9 e manutenção da cadência de pedalada entre 70 e $110 \mathrm{rpm}$. A exaustão foi definida como o instante no qual os ciclistas não fossem mais capazes de manter a cadência de $70 \mathrm{rpm}$. $\mathrm{OVO}_{2 \text { Max }}$ foi definido como o maior valor mensurado de $\mathrm{VO}_{2}$ no último minuto do teste ${ }^{10}$. $\mathrm{O}$ consumo de oxigênio $\left(\mathrm{VO}_{2}\right)$ e a produção de dióxido de carbono $\left(\mathrm{VCO}_{2}\right)$ foram monitorados a cada respiração, utilizando um sistema de ergoespirometria de circuito aberto (MGC CPX/D, Medical Graphics Corp., St Louis, EUA). Anteriormente a coleta de dados de cada ciclista, o analisador de gases foi calibrado utilizando uma me- dida de volume de ar conhecida e concentrações de $\mathrm{O}_{2}$ e $\mathrm{CO}_{2}$ conhecidas. Todos os sujeitos receberam incentivo verbal durante a realização do teste para que obtivessem o melhor desempenho no mesmo. De forma paralela, a potência produzida foi continuamente mensurada, permitindo a determinação do pico de potência produzido no teste. $\mathrm{O}$ segundo limiar ventilatório foi determinado utilizando-se o critério do equivalente ventilatório por meio de dois avaliadores experientes ${ }^{11}$.

Avaliação da mudança na posição dos joelhos No segundo dia de avaliação, os ciclistas retornaram ao laboratório para serem avaliados, utilizando suas próprias bicicletas, sendo estas acopladas a um ciclo simulador Cateye CS1000 (Cateye CO., Osaka, Japão).

Duas hastes metálicas foram posicionadas póstero-anteriormente, permanecendo paralelas ao quadro da bicicleta de cada ciclista. Estas hastes foram posicionadas com o intuito de prover uma referência para que os ciclistas pudessem tangenciar as mesmas com a parte lateral dos joelhos durante o ciclo de pedalada na situação em que deveriam afastar os joelhos do quadro da bicicleta. As hastes metálicas não foram utilizadas nas demais posições avaliadas, uma vez que a posição de referência era a usualmente adotada, e a posição de adução envolvia um movimento dos joelhos próximo ao quadro da bicicleta.

Três posições foram avaliadas:

- Posição de Referência: aquela em que os ciclistas mantiveram os joelhos na distância preferida em relação ao quadro da bicicleta, a qual era usualmente utilizada em treinamentos e competições;

- Posição de Adução: aquela em que os ciclistas mantiveram os joelhos tangenciando o quadro da bicicleta, mantendo a adução dos quadris durante o ciclo de pedalada;

- Posição de Abdução: aquela em que os ciclistas tangenciavam as hastes metálicas, descritas anteriormente, com os joelhos, durante o ciclo de pedalada. A distância entre a haste metálica e o eixo central do quadro da bicicleta foi padronizada em $30 \mathrm{~cm}$ para todos os ciclistas.

Na Figura 2, é apresentada uma imagem de um ciclista durante a coleta de dados, do segundo dia de avaliação, com a finalidade de representar a diferença nas posições dos joelhos em relação ao quadro da bicicleta nas posições de Adução e Abdução. 


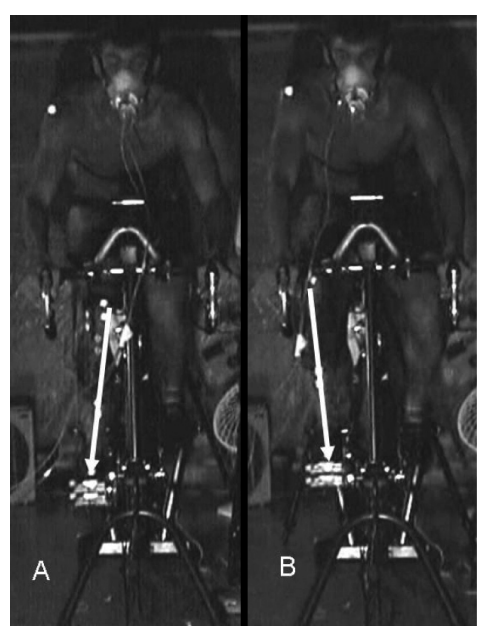

Figura 2. Ilustração das posições de Adução (A) e Abdução (B), indicando a diferença na posição dos joelhos dos ciclistas em relação ao quadro da bicicleta. Na figura, é representado o vetor que compreende a aplicação de força estimada no plano frontal em cada uma das posições por meio da análise qualitativa do alinhamento do segmento perna do ciclista apresentado.

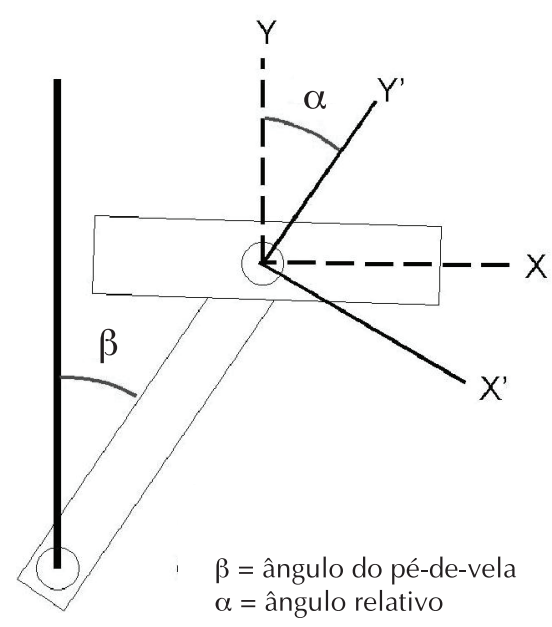

Figura 3. Diagrama dos ângulos do pé-de-vela ( $\beta$ ) e do peda relativo à posição do pé-de-vela $(\alpha)$. (Carpes et al.13).

\section{Determinação da carga de trabalho}

A carga de trabalho do segundo dia de avaliação foi controlada por meio da fixação das marchas da bicicleta de cada ciclista, sendo esta ajustada durante o período de aquecimento que antecedeu a coleta dos dados. $\mathrm{O}$ valor do $\mathrm{VO}_{2}$ referente ao segundo limiar ventilatório individual ${ }^{8}$ foi selecionado e utilizado como valor de referência para a determinação da relação de marchas, quando o ciclista pedalava com os joelhos na Posição de Referência durante o período de aquecimento. Estes utilizaram a cadência preferida de pedalada durante a determinação da relação de marchas, sendo esta possível de ser modificada com o intuito de manter o $\mathrm{VO}_{2}$ nas demais posições avaliadas. $\mathrm{O} \mathrm{VO}_{2}, \mathrm{VCO}_{2}$ e a taxa de troca respiratória (RER) foram obtidos a cada respiração pelo mesmo sistema de ergoespirometria descrito anteriormente. Nas condições determinadas, todos os ciclistas pedalaram por três minutos após a estabilização do $\mathrm{VO}_{2}$, sendo coletados, durante os 30 segundo finais, a força aplicada no pedal, as variáveis angulares do pedal, assim como as variáveis respiratórias de cada ciclista ${ }^{12}$.

\section{Aquisição dos sinais}

Um pedal bi-dimensional instrumentado com strain gages foi utilizado para mensurar as componentes normal ( $\mathrm{Fy}$ ) e tangencial ( $\mathrm{Fx}$ ) da força aplicada pela perna direta dos ciclistas ${ }^{8}$. Os sinais de força foram amplificados (ganho de $1 \mathrm{~K}$ ) por um condicionador de sinais Entran MSC6. Os ciclos de pedalada foram determinados por meio de um sensor eletromagnético (reed switch) acoplado ao quadro da bicicleta de cada ciclista ${ }^{3}$. O ângulo do pedal em relação à posição do pé-de-vela foi mensurado por meio de um potenciômetro Spectrol $2 \mathrm{~K} \Omega^{6,13}$.

Os sinais das componentes Fy e Fx, assim como do reed switch e do potenciômetro foram digitalizados por meio de um conversor analógico-digital com resolução de 16 bits (DI-720, Dataq Instruments, EUA). Todos os sinais foram adquiridos com o uso do software Windaq (Windaq, Dataq Instruments, EUA) de 16 canais, com freqüência de amostragem de 2,3 $\mathrm{KHz}$ por canal.

\section{Processamento dos sinais}

A média e o desvio padrão do $\mathrm{VO}_{2}$ foram calculados para cada uma das posições avaliadas com o objetivo de estimar o custo metabólico.

As componentes da força aplicada no pedal, assim como os sinais do reed switch e do potenciômetro foram processados, utilizando rotinas desenvolvidas no software MATLAB ${ }^{\circledR}$ (MathWorks Inc., EUA). Estes sinais foram filtrados, utilizando-se um filtro digital passa baixa do tipo Butterworth de $3^{2}$ ordem, com freqüência de corte de $10 \mathrm{~Hz}$. Todas as variáveis foram obtidas por meio da análise dos dez primeiros ciclos de pedalada.

Para o cálculo da força efetiva, um modelo bi-dimensional foi assumido, no qual apenas as componentes da força atuantes no plano sagital foram consideradas, sendo Fy e Fx decompostas em relação ao pé-de-vela. O cálculo da força efetiva consistiu, então, do somatório dos componentes de Fy e Fx perpendiculares ao pé-de-vela, utilizando-se o ângulo do pedal relativo à posição do pé-de-vela, como apresentado na equação $1^{13}$. 
Tabela 1. Resultados do consumo de oxigênio $\left(\mathrm{VO}_{2}\right)$, potência produzida (PO) e cadência de pedalada dos seis ciclistas nas três posições avaliadas (Referência, REF; Adução, ADU e Abdução, ABD). Os resultados são apresentados em relação à média e desvio padrão (DP).

\begin{tabular}{|c|c|c|c|c|c|c|c|c|c|}
\hline \multirow{2}{*}{ Ciclista } & \multicolumn{3}{|c|}{$\mathrm{VO}_{2}\left(\mathrm{ml} \cdot \mathrm{kg}^{-1} \cdot \mathrm{min}^{-1}\right)$} & \multicolumn{3}{|c|}{$\mathrm{PO}(\mathrm{W})$} & \multicolumn{3}{|c|}{ Cadência (rpm) } \\
\hline & REF & ADU & $\mathrm{ABD}$ & REF & ADU & $\mathrm{ABD}$ & REF & ADU & $\mathrm{ABD}$ \\
\hline A & 62,1 & 63,4 & 61,9 & 253 & 275 & 218 & 93 & 97 & 95 \\
\hline B & 50 & 51,1 & 51,9 & 331 & 278 & 289 & 93 & 95 & 95 \\
\hline C & 46,8 & 43,3 & 44,7 & 197 & 221 & 285 & 75 & 79 & 93 \\
\hline $\mathrm{D}$ & 46,9 & 46,6 & 47,8 & 208 & 234 & 172 & 90 & 93 & 91 \\
\hline $\mathrm{E}$ & 46,9 & 47,5 & 49,0 & 236 & 275 & 281 & 83 & 95 & 92 \\
\hline $\mathrm{F}$ & 49,7 & 50,4 & 49,2 & 252 & 298 & 253 & 99 & 107 & 107 \\
\hline Média & 50,4 & 50,4 & 50,8 & 246 & 263 & 250 & 89 & $94 *$ & $95^{*}$ \\
\hline DP & 5,9 & 6,9 & 5,9 & 47 & 29 & 46 & 8 & 8 & 5 \\
\hline
\end{tabular}

* Diferença significativa em relação à posição de referência $(p<0,05)$.

\# Diferença significativa entre as posições de adução e abdução $(p<0,05)$.

$$
F E=F y^{\perp}+F x^{\perp}
$$

Equação 1. Cálculo da força efetiva (FE), no qual $F y^{\perp}$ representa o componente de Fy que é perpendicular ao pé-de-vela, e $\mathrm{Fx}^{\perp}$ representa o componente de Fx que é perpendicular ao pé-de-vela.

Os valores positivos da força efetiva foram considerados como capazes de gerar torque no sentido do movimento do pé-de-vela. A força resultante (FR) aplicada no pedal foi calculada por meio da equação $2^{8}$.

$$
F R=\sqrt{F y^{2}+F x^{2}}
$$

Equação 2. Cálculo da força resultante (FR).

$O$ índice de efetividade (IE) foi calculado por meio da equação 3, sendo expresso em valores percentuais do impulso linear gerado pela FR, de acordo com LaFortune \& Cavanagh ${ }^{14}$.

$I E=\int_{0}^{360} F E d t / \int_{0}^{360} F R d t$

Equação 3. Cálculo do índice de efetividade (IE), no qual FE representa a força efetiva e FR representa a força resultante.

A potência produzida $(\mathrm{PO})$ foi determinada como o produto do torque e da velocidade angular, como indicado na equação 4 . $O$ torque foi mensurado como o produto da FE e do tamanho do pé-de-vela, enquanto a velocidade angular foi obtida pela cadência de pedalada

$P O=2 \cdot T \cdot \omega$

Equação 4. Cálculo da potência produzida (PO), no qual T representa o torque produzido pelo pedal direito e $\omega$ representa a velocidade angular do pé-de-vela.

\section{Análise estatística dos dados}

Estatística descritiva foi utilizada para a apresenta- ção dos resultados em relação a sua média e desviopadrão. A normalidade dos dados foi verificada por meio do teste de Shapiro Wilk. Em caso de distribuição normal dos dados, a análise de variância para medidas repetidas (ANOVA One way) foi aplicada para comparação do $\mathrm{VO}_{2}$, cadência de pedalada, valores médios de FE e FR, IE e PO nas três posições avaliadas. Post Hoc de Tukey HSD foi utilizado para identificação das diferenças quando a ANOVA indicava interação. No caso de não haver distribuição normal dos dados, o teste de Fridman foi aplicado para identificação da ocorrência ou não de diferenças, seguido do teste de Wilcoxon, com o objetivo de identificar entre quais posições estas diferenças ocorreram. Para todos os procedimentos estatísticos, o pacote estatístico SPSS versão 12.0 (SPSS Inc., USA) foi utilizado com nível de significância de 0,05.

\section{RESULTADOS}

Os resultados individuais do $\mathrm{VO}_{2}, \mathrm{PO}$ e cadência de pedalada dos seis ciclistas, assim como os valores médios do grupo são apresentados na tabela I.

Os resultados do $\mathrm{VO}_{2}$ indicam que o custo metabólico dos ciclistas não diferiu significativamente entre as posições avaliadas. A cadência de pedalada foi significativamente menor na Posição de Referência, comparada às posições de Adução e Abdução.

As componentes da força aplicada no pedal, Fy e Fx, são apresentadas na figura 4, juntamente com a força efetiva (FE) e a força resultante (FR) nas três posições avaliadas, com o objetivo de representar o padrão de aplicação dos ciclistas avaliados.

Os valores médios da FE e FR, assim como os resultados do índice de efetividade (IE) são apresentados na tabela II. 

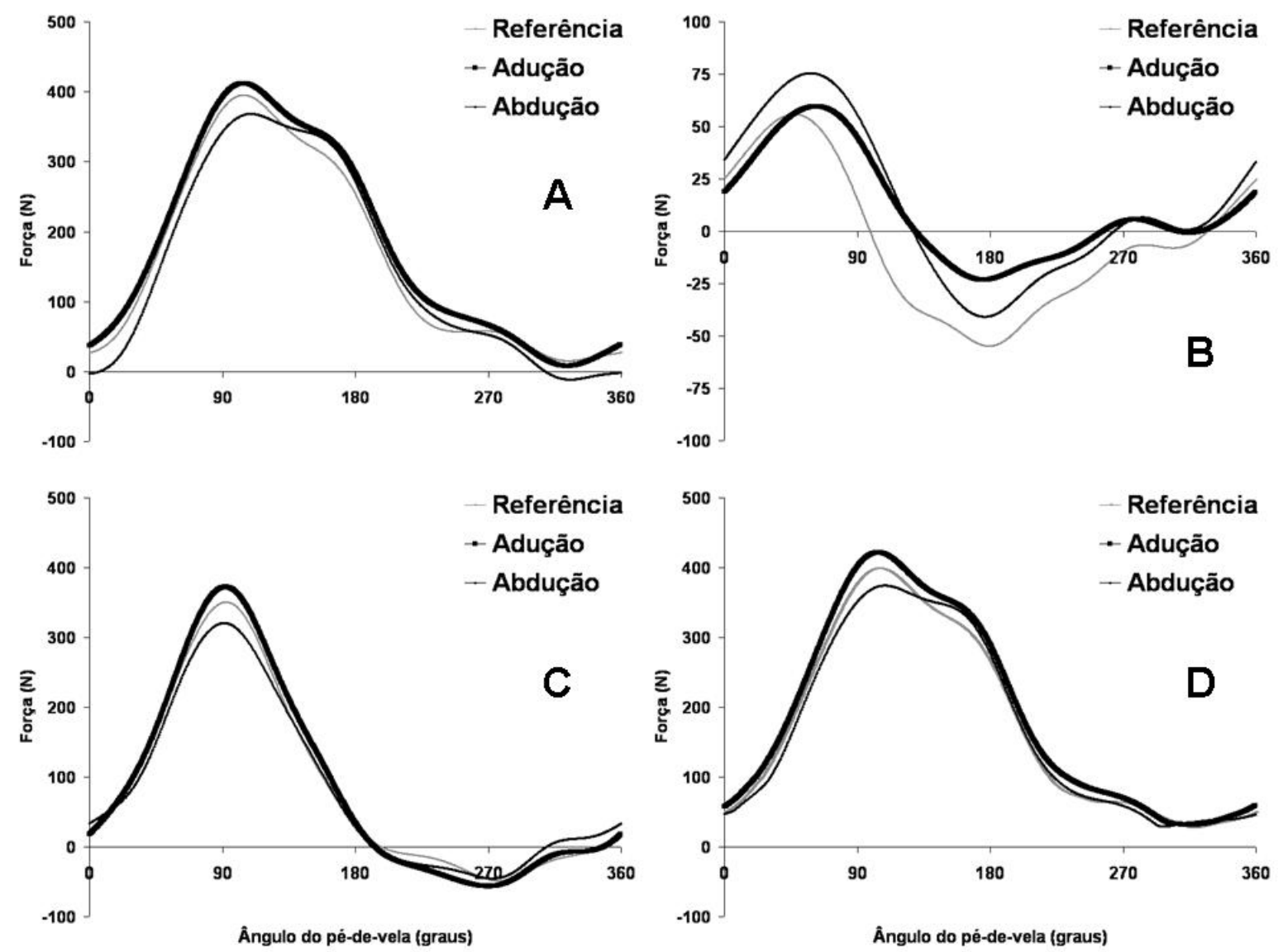

Figura 4. As componentes normal (A) e tangencial (B) da força aplicada no pedal, assim como a força efetiva (C) e a força resultante (D) média dos seis ciclistas ao longo do ciclo de pedalada para cada posição avaliada.

Tabela 2. Resultados do valor médio da FE e FR dos dez ciclos de pedalada analisados, assim como do IE. Estes são apresentados individualmente para cada ciclista e por meio da média e desvio padrão (DP) do grupo nas três posições avaliadas (Referência, REF; Adução, ADU e Abdução, ABD).

\begin{tabular}{lccccccccc}
\hline Ciclista & \multicolumn{3}{c}{ FE $(\mathrm{N})$} & \multicolumn{3}{c}{ FR $(\mathrm{N})$} & & \multicolumn{2}{c}{ IE $(\%)$} \\
\hline A & REF & ADU & ABD & REF & ADU & ABD & REF & ADU & ABD \\
B & 118,52 & 129,17 & 102,33 & 299,08 & 298,11 & 306,39 & 49 & 51 & 44 \\
C & 150,64 & 131,38 & 131,82 & 278,32 & 333,00 & 347,73 & 60 & 50 & 48 \\
D & 89,65 & 100,87 & 129,86 & 216,27 & 230,35 & 251,62 & 50 & 50 & 52 \\
E & 94,80 & 106,47 & 78,64 & 217,16 & 234,00 & 197,70 & 50 & 49 & 46 \\
F & 107,68 & 125,21 & 128,18 & 265,76 & 327,85 & 275,34 & 48 & 47 & 51 \\
Média & 118,42 & 139,51 & 118,60 & 204,56 & 283,72 & 244,51 & 62 & 56 \\
DP & 113,29 & 122,10 & 114,91 & 246,86 & $284,50 *$ & 270,55 & 53 & 51 \\
\hline
\end{tabular}

* Diferença significativa em relação à posição de referência $(p<0,05)$.

\# Diferença significativa entre as posições de adução e abdução $(p<0,05)$.

A FE não apresentou diferenças significativas entre as três posições avaliadas, no entanto, a FR foi significativamente maior na posição de Adução quando comparada à posição de Referência. $\mathrm{O}$ IE não apresentou diferenças significativas entre as três posições avaliadas.

\section{DISCUSSÃO}

O objetivo do presente estudo foi comparar a força aplicada no pedal quando os ciclistas pedalaram com os joelhos tangenciando o quadro da bicicleta (Posição de Adução), em relação à sua posição usual de pedalada (Posição de Referência) e a posição na qual os ciclistas pedalavam com os joelhos o mais afastado possível do quadro da bicicleta (Posição de Abdução). Os resultados encontrados no presente estudo vão de encontro à hipótese formulada, de que mudanças na posição dos joelhos em relação ao quadro da bicicleta (posições de Adução e 
Abdução), devido à mudança no ângulo do quadril, poderiam reduzir a força aplicada no pedal, comparado à Posição de Referência. Observou-se manutenção do índice de efetividade com a mudança nas posições dos joelhos, indicando que a posição dos joelhos no plano frontal parece não afetar o aproveitamento da força aplicada no pedal. Na posição de Adução, observou-se um aumento da força resultante aplicada no pedal, o que indica uma maior aplicação de força nesta posição.

$\mathrm{O}$ controle do $\mathrm{VO}_{2}$ foi importante para a rejeição da hipótese de que a intensidade do exercício pudesse afetar a força aplicada no pedal. A escolha da intensidade de exercício correspondente ao segundo limiar ventilatório foi feita com o intuito de simular um nível de esforço vivenciado pelos ciclistas em competições ${ }^{15}$.

A hipótese de que poderia ocorrer uma redução na efetividade da força aplicada no pedal nas posições de Adução e Abdução não foi suportada no presente estudo, uma vez que a FE e o IE não apresentaram diferenças significativas entre as posições avaliadas. $\mathrm{O}$ aumento significativo da FR na posição de Adução indica uma melhor condição para aplicação de força no pedal nesta posição. Burke e Pruitt ${ }^{5}$ reportam que alguns ciclistas frequentemente assumem a posição dos joelhos tangenciando o quadro da bicicleta durante o ciclo de pedalada com o objetivo de reduzir a área frontal e conseqüentemente, minimizar a força de arrasto. Esta estratégia, no entanto, parece não afetar a capacidade técnica do ciclista em direcionar a força aplicada no pedal, podendo proporcionar uma melhor condição para aplicação de força (aumento da FR).

Para a manutenção dos joelhos próximos ao quadro da bicicleta, um aumento no ângulo de adução do quadril é observado de forma qualitativa, o que pode aumentar o comprimento dos músculos responsáveis pela rotação externa da articulação do quadril (ex. gluteus maximus). O maior comprimento destes músculos poderia permitir uma melhor capacidade de produção de força por parte destes músculos, aumentando a produção de torque extensor no quadril na fase de propulsão da pedalada ${ }^{16}$. Este aumento no torque extensor na articulação do quadril justificaria o aumento da FR observado na posição de Adução. No entanto, os ciclistas do presente estudo não foram capazes de direcionar de forma adequada esta maior quantidade de força produzida durante a fase de propulsão, indicando que o aumento na geração de força resultante não reflete um aumento na potência produzida.

As posições de Adução e Abdução alteram a posição dos joelhos em relação ao eixo do pedal no plano frontal, como representado na Figura 2, o que poderia alterar a linha de ação do músculo rectus femoris. Van Ingen Schenau et al. ${ }^{17}$ e Hof ${ }^{18}$ indicam que o músculo rectus femoris apresenta função importante no direcionamento da força produzida pelos músculos mono-articulares extensores do quadril e do joelho durante movimentos multi-articulares.

A posição de Abdução foi avaliada com o intuito de simular uma posição não-convencional no ciclismo, na qual os joelhos eram mantidos o mais afastado possível do quadro da bicicleta. Esta posição não é comumente adotada, visto que pode resultar em um aumento da área frontal e consequente aumento da força de arrasto. No entanto, a hipótese de que esta posição seria prejudicial para o aproveitamento das forças aplicadas foi refutada perante a similaridade estatística da FE e do IE nesta posição, quando comparada às posições de Referência e Adução. A magnitude da força aplicada no pedal, mensurada por meio da FR, parece não ter sido afetada pela adoção da posição de $\mathrm{Ab}$ dução. Estes resultados não eram esperados, visto que os ciclistas reportaram a posição de Abdução como sendo desconfortável quando comparada às posições de Referência e Adução.

$O$ fato de que os ciclistas pudessem alterar a cadência de pedalada nas posições de Adução e Abdução resultou em aumento da mesma quando comparadas à posição de Referência. Esta escolha foi feita com o intuito de manutenção do $\mathrm{VO}_{2}$, visto que a fixação da cadência poderia resultar em um aumento do custo energético com a mudança da posição dos joelhos. $\mathrm{O}$ aumento da cadência está comumente relacionado com reduções na FR, FE e no $\mathrm{IE}^{8}$. Faria ${ }^{19}$ indica que a cadência preferida dos ciclistas permanece em torno de 90 e 100 rpm, o que foi observado nas posições de Adução e Abdução. Devido às possíveis mudanças no comprimento de alguns músculos (ex. adductor longus e gluteus maximus) quando foi alterada a posição dos joelhos em relação ao quadro da bicicleta, os ciclistas aumentaram a cadência de pedalada com o objetivo de manter a capacidade de produção de força e reduzir a sobrecarga muscular ${ }^{20}$. A menor cadência de pedalada na posição de Referência pode estar relacionada com uma adaptação dos ciclistas ao comprimento muscular imposto pela posição dos joelhos resultante desta condição, e é mais utilizada em treinamentos e competições ${ }^{21,22}$. Duc et al. ${ }^{10}$ indicam que o controle do esforço no ciclismo está altamente relacionado com um 
"estado estável muscular", que também justificaria o aumento na cadência nas posições de Adução e Abdução com o objetivo de manutenção do $\mathrm{VO}_{2}$. A análise da ativação muscular nestas posições auxiliaria no melhor entendimento do grau de recrutamento solicitado pelo sistema nervoso central aos músculos do membro inferior com a mudança na posição dos joelhos.

Características posturais dos membros inferiores resultantes de aspectos morfológicos distintos dos ossos e músculos, assim como demandas funcionais das atividades diárias, podem alterar a capacidade de produção de força nos diferentes comprimentos musculares ${ }^{21,22}$. A mobilidade da articulação do tornozelo, que apresenta graus de liberdade nos três eixos ortogonais, deve ser considerada um fator interveniente no presente estudo, sendo necessária a sua mensuração em avaliações futuras. A relação das características antropométricas e da amplitude de movimentos da articulação do tornozelo pode influenciar o padrão de aplicação de força nos pedais pelos ciclistas.

Dentre as limitações do presente estudo, destaca-se a impossibilidade de mensurar a componente médio-lateral da força aplicada no pedal. $O$ pedal instrumentado utilizado no presente estudo permitia apenas a medição das componentes normal e tangencial da força aplicada no pedal. Esta, provavelmente, apresentaria sensibilidade às alterações conduzidas no presente estudo. Outra limitação foi a impossibilidade de mensurar as variáveis cinemáticas de forma tridimensional. Esta resultou de problemas técnicos no sistema de videogrametria durante a coleta de dados, o que impediu a verificação da movimentação do joelho nos planos frontal e sagital, permitindo-se a compreensão das compensações articulares quando alterada a posição do joelho no plano frontal.

Com isto, sugere-se que avaliações posturais sejam conduzidas de forma paralela às avaliações biomecânicas de ciclistas. Estudos futuros devem ser conduzidos associando além da avaliação postural dos ciclistas, a análise de variáveis cinemáticas tridimensionais com a avaliação de um grupo maior de ciclistas. Para fins de descrição do comportamento da força aplicada no pedal com a mudança na posição dos joelhos, o modelo de estudo preliminar adotado foi satisfatório.

\section{CONCLUSÃO}

A mudança na posição dos joelhos em relação ao quadro da bicicleta parece não afetar a técnica de pedalada de ciclistas. No entanto, na posição de Adução foi possível observar um aumento na capacidade de aplicação de força no pedal. Estes resultados indicaram que os ciclistas foram capazes de aplicar mais força no pedal na posição de Adução, no entanto, não foram capazes de melhorar o aproveitamento desta força. $\mathrm{O}$ treinamento na posição de Adução é sugerido para que ocorra uma adaptação muscular nessa posição e posterior melhora da técnica de pedalada, resultando, possivelmente, em um aproveitamento aumentado da força aplicada no pedal, visto que esta posição pode melhorar aspectos aerodinâmicos da pedalada 5 . Uma repercussão negativa da adoção da posição de Adução seria a sobrecarga sobre as estruturas mediais do joelho ${ }^{7}$, esta devendo ser avaliada de forma combinada à análise das características posturais do ciclista. A avaliação individual de cada ciclista permite, com isto, melhores definições quanto a posição dos joelhos a ser adotada durante a pedalada.

\section{REFERÊNCIAS BIBLIOGRÁFICAS}

1. Hagberg J, McCole S. Energy expenditure during cycling. In: Burke ER. editor. High-tech Cycling 1996; Champaign, IL, Human Kinetics. p. 167-184.

2. McCole SD, Claney K, Conte JC, Anderson R, Hagberg JM. Energy expenditure during bicycling. J Appl Pysiol 1990;68(2):748-753.

3. Welbergen E, Clijsen LPVM. The influence of body position on maximal performance in cycling. Euro J Appl Physiol 1990;61(1):138-142.

4. Olds T, Olive S. Methodological considerations in the determination of projected frontal area in cyclists. J Sports Sci 1999;17(4):335-345.

5. Burke ER, Pruitt AL. Body positioning for cycling. In: Burke ER. editor. High Tech Cycling 2003; Champaign, IL, Human Kinetics. p 69-92.

6. Hull ML, Davis RR. Measurement of pedal loading in bicycling: I. Instrumentation. J Biomech 1981;14(12):843-856.

7. Ericson MO, Nisell R, Ekholm J. Varus and valgus loads on the knee joint during ergometer cycling. Scand J Sports Sci 1984;6(2):39-45.

8. Candotti CT, Ribeiro J, Soares DP, Reischak AO, Loss JF, Guimarães AC. Effective force and economy of triathletes and cyclists. Sports Biomech 2007;6(1):31-43.

9. Lucia A, Hoyos J, Perez M, Santalla A, Chicharro J. Inverse relationship between VO2max and economy/ efficiency in world-class cyclists. Med Sci Sport Exerc 2002;34(12):2079-2084.

10. Duc S, Betik AC, Grappe F. EMG activity does not change during a time trial in competitive cyclists. Int J Sport Med 2005;26(2):145-150.

11. Amann M, Subuhi A.W, Foster C. Predictive validity of ventilatory and lactate thresholds for cycling 
time trial performance. Scand J Med Sci Sport 2006;16(1):27-34

12. Diefenthaeler F, Bini RR, Nabinger E, Laitano OL, Carpes FP, Mota CB, Guimarães ACS. Proposta metodológica para a avaliação da técnica da pedalada de ciclistas: estudo de caso. Rev Bras Med Esporte 2008;14(2):145-148.

13. Carpes FP, Bini RR, Nabinger E, Diefenthaeler F, Mota $\mathrm{CB}$, Guimarães ACS. Aplicação de força no pedal em prova de ciclismo $40 \mathrm{~km}$ contra-relógio: estudo preliminar. Rev Bras Educ Fís Esp 2005;19(2):105-13.

14. LaFortune MA, Cavanagh PR. Effectiveness and Efficiency during bicycle riding. In: Matsui, Kobashi K. editor. Biomechanics VIII-B; Champaign, IL, Human Kinetics. 1983; p. 167-184.

15. Amann M, Subuhi A, Foster C. Influence of testing protocol and ventilatory threshold and cycling performance. Med Sci Sports Exerc 2004;36(4):613-622.

16. Kautz SA, Neptune RR. Biomechanical determinants of pedaling energetics: internal and external work are not indepent. Exerc Sports Sci Rev 2002;30(4):159-165.

17. Van Ingen Schenau GJ, Pratt CA, MacPherson JM. Differential use of control of mono- and biarticular muscle. Hum Mov Sci 1994;13(3):495-517.

18. Hof AL. The force resulting from the action of mono- and biarticular muscles in a limb. J Biomech 2001;34(8):1085-1089.

19. Faria IE. Energy expenditure, aerodynamics and medical problems in cycling. Sports Med 1992;14(1):43-63.

20. Marsh AP, Martin PE, Sanderson DJ. Is a joint momentbased cost function associated with preferred cycling cadence? J Biomech 2000;33(2):173-180.
21. Herzog W, Guimarães ACS, Anton MG, CarterErdman KA. Moment-length relations of rectus femoris muscles of speed skaters/cyclists and runners. Med Sci Sports Exerci 1991;23(11): 1289-1296.

22. Savelberg HHCM, Meijer K. Contribution of mono- and biarticular muscles to extending knee joint moments in runners and cyclists. J Appl Physiol 2003;94(6):2241-2248.

\section{Agradecimentos}

Os autores dedicam este trabalho a memória do "pai" e professor Antônio Carlos Stringhini Guimarães pela sua amizade, ensinamentos e pela concepção deste projeto. $\mathrm{O}$ professor Guimarães foi o idealizador deste projeto, no entanto o seu falecimento o impediu de participar da conclusão do mesmo.

\section{Endereço para correspondência}

Rodrigo Rico Bini

Institute of Sport and Recreation Research New

Zealand, room AH221D

School of Sport and Recreation

AUT University

90 Akoranga Drive, Northcote

North Shore City, Auckland 0637

New Zealand

Email: bini.rodrigo@gmail.com 